\title{
Clinical and prognostic significance of miR-155 and miR-146a expression levels in formalin-fixed/paraffin-embedded tissue of patients with diffuse large B-cell lymphoma
}

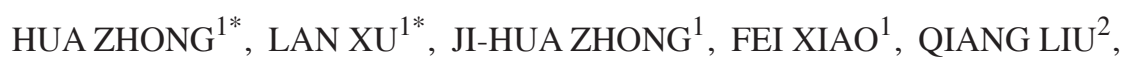 \\ HONG-HUI HUANG ${ }^{1}$ and FANG-YUAN $\mathrm{CHEN}^{1}$ \\ Departments of ${ }^{1}$ Hematology, and ${ }^{2}$ Pathology, Renji Hospital, Shanghai Jiaotong University, \\ School of Medicine, Shanghai 200127, P.R. China
}

Received January 5, 2012; Accepted January 30, 2012

DOI: $10.3892 /$ etm.2012.502

\begin{abstract}
It has been found that aberrant expression of microRNAs (miRNAs) is strongly associated with carcinogenesis. In the present study, we investigated the expression of miR-155 and miR-146a in diffuse large B-cell lymphoma (DLBCL) patients $(n=90)$. The expression levels of miR-155 and miR-146a were significantly higher in de novo DLBCL patients. miR-146a expression levels were associated with miR-155, lactate dehydrogenase, $\beta 2$ microglobulin, c-myc, International Prognostic Index status and Eastern Cooperative Oncology Group performance status. We found that patients with low miR-155 and miR-146a expression levels achieved a higher complete remission rate, higher overall response rate and longer progression-free survival time. Moreover, a high expression level of miR-155, but not miR-146a, was an independent indicator for chemotherapy protocol selection in our study. Patients with high expression of miR-155 received more survival benefits from rituximab treatment. These data suggest that miR-155 and miR-146a have potential as diagnostic and prognostic markers in DLBCL.
\end{abstract}

\section{Introduction}

MicroRNAs (miRNAs) are endogenous, single-stranded noncoding RNAs that consist of approximately 22 nucleotides. They play important regulatory roles at the post-transcriptional level by binding to their targeted mRNAs. They either block their translation or initiate their degradation, according to the

Correspondence to: Dr Fang-Yuan Chen, Department of Hematology, Renji Hospital, Shanghai Jiaotong University, School of Medicine, No. 1630 Dong Fang Road, Shanghai 200127, P.R. China

E-mail: chenfy04@yahoo.com.cn

*Contributed equally

Key words: diffuse large B-cell lymphoma, miR-155, miR-146a, diagnostic and prognostic marker degree of complementarity with their targets (1). In addition to controling hematopoiesis, miRNAs play key regulatory roles in a diverse range of pathways, including developmental timing, cell differentiation, apoptosis, cell proliferation and organ development (2). Direct involvement of miRNAs in cancer has been suggested by studies demonstrating that several miRNAs are localized in genomic regions associated with cancer, such as breakpoint regions in chromosome aberrations involving oncogenes or tumor-suppressor genes, minimal regions of loss of heterozygosity, minimal regions of amplification, and at loci close to fragile sites and integration sites of the human papillomavirus (3). miRNAs are proposed to play a direct role in oncogenesis, as they can function as both oncogenes and tumor-suppressor molecules. Currently, at least three mechanisms are understood whereby miRNAs are deregulated in cancer: i) chromosomal lesions at regions encoding microRNAs, ii) defects in the miRNA biosynthetic pathway machinery, and iii) epigenetic regulation (4). miRNAs also have been demonstrated to have diagnostic, prognostic and therapeutic potential in cancer (5-7).

Diffuse large B-cell lymphoma (DLBCL) is the most common adult lymphoma accounting for nearly $40 \%$ of all lymphoid tumors (8). Gene expression and immunohistochemical studies of this clinically heterogeneous disease have revealed the presence of at least two distinct subtypes of DLBCL: germinal centre B cell-like (GCB) and non-germinal centre-like (non-GCB) $(9,10)$. A hallmark of non-GCB DLBCL biology is the constitutive activation of the NF- $\kappa B$ pathway, which promotes proliferation and differentiation and suppresses apoptosis (11). miR-155 and miR-146a are located on chromosome 21q21 and 5q33 dividedly. These two miRNAs are both thought to be regulated by NF- $\mathrm{BB}(12-14)$.

Formalin-fixed/paraffin-embedded (FFPE) tissue samples are the most readily available archival material. They represent an invaluable source for the study of human disease. In contrast to traditional gene expression studies that require difficult to obtain freshly frozen clinical material, miRNAs can be successfully isolated from routinely processed formalin-fixed material, because of their relative resistance to RNase degradation and their small size. Such samples, which have been matched to frozen tissue, give remarkably similar results by 
real-time quantitive PCR (RTQ-PCR) and microarray analysis $(15,16)$.

Despite new drugs, such as rituximab, that have been used in treatment regimes, the majority of patients succumb to this aggressive disease, especially those with the non-GCB subtype (17). Thus, it is necessary to discover new potential diagnostic and prognostic markers for clinical use. In this study, we investigated the expression levels of miR-155 and miR-146a in FFPE tissue samples of DLBCL patients. A correlation between miR-155 and miR-146a expression levels and patient outcomes (complete remission rate, overall response rate and prognosis-free survival) with DLBCL subtypes was found by multivariate analysis.

\section{Materials and methods}

Patient samples. FFFPE biopsy samples of 90 of de novo DLBCL cases and 31 reactive hyperplasia lymphoid node cases (16 males, 15 females; median age 45.3 years) were obtained from the Pathology Department of Renji Hospital. All samples were collected at the time of initial diagnosis (prior to treatment). A summary of DLBCL de novo patient details are provided in Table I. Informed consent was provided according to the Declaration of Helsinki. The study protocol was approved by the Medical Ethics Committee of Renji Hospital.

Immunohistochemical staining. DLBCL de novo cases were classified immunohistochemically as GCB- or non-GCB-type using monoclonal antibodies against CD10, BCL6 and melanoma-associated antigen (mutated) 1 (MUM1) as previously described (15). Monoclonal antibody against c-myc (Shanghai Long Island Biotech, Shanghai, China) was also used to examine the protein expression. Relevant ethical permission was obtained for the use of all samples.

$R N A$ purification and RTQ-PCR. Total RNA was purified from $4 \times 20 \mu \mathrm{m}$ FFPE sections using the Recoverall kit from Ambion (Huntingdon, UK) in accordance with the manufacturer's instructions. TaqMan miRNA assays were used to detect and quantify mature miR-155 and miR-146a as previously described (18) using TaqMan ${ }^{\circledR}$ MicroRNA Reverse Transcription kit (4366597; Applied Biosystems, Foster City, CA, USA) and PCR 9700 sequence detection system (Applied Biosystems). Normalization was performed with U48. RTQ-PCR was performed in triplicate, including no-template controls. The $2^{-\Delta \mathrm{Ct}}$ method was used in the analysis of PCR data $[\Delta \mathrm{Ct}=$ mean $\mathrm{Ct}$ (microRNA of interest) - mean $\mathrm{Ct}$ (U48)].

Chemotherapy protocol. De novo patients with DLBCL were treated with the CHOP (cyclophosphamide $750 \mathrm{mg} / \mathrm{m}^{2}$ on day 1 , vincristine $1.4 \mathrm{mg} / \mathrm{m}^{2}$ on day 1 , epirubicin $60 \mathrm{mg} / \mathrm{m}^{2}$ on day 1 and prednisone $60 \mathrm{mg} / \mathrm{m}^{2}$ on days $1-5$ ) or R-CHOP (rituximab $375 \mathrm{mg} / \mathrm{m}^{2}$ on day 0 , cyclophosphamide $600 \mathrm{mg} / \mathrm{m}^{2}$ on day 1 , vincristine $1.4 \mathrm{mg} / \mathrm{m}^{2}$ on day 1 , epirubicin $60 \mathrm{mg} / \mathrm{m}^{2}$ on day 1 and prednisone $60 \mathrm{mg} / \mathrm{m}^{2}$ on days 1-5) regimen.

Statistical and survival analysis. miRNA expression levels and clinicopathological parameters were analyzed using SPSS 16.0 (SPSS Inc., Chicago, IL, USA). The $\chi^{2}$ test, the Mann-Whitney $\mathrm{U}$ test, Kaplan-Meier survival analysis and multivariate Cox
Table I. Patient characteristics at initial diagnosis $(n=90)$.

\begin{tabular}{|c|c|c|c|c|}
\hline $\begin{array}{l}\text { Clinical } \\
\text { feature }\end{array}$ & No. & $\begin{array}{l}\text { GCB } \\
\mathrm{n}(\%)\end{array}$ & $\begin{array}{c}\text { non-GCB } \\
\text { n (\%) }\end{array}$ & P-value \\
\hline Age (years) & & & & 0.222 \\
\hline$<60$ & 54 & $15(71.4)$ & $39(56.5)$ & \\
\hline$\geq 60$ & 36 & $6(28.6)$ & $30(43.5)$ & \\
\hline Gender & & & & 0.689 \\
\hline Female & 48 & $12(57.1)$ & $36(52.2)$ & \\
\hline Male & 42 & 9 (42.9) & $33(47.8)$ & \\
\hline Stage & & & & 0.363 \\
\hline I & 9 & $4(19.0)$ & $5(7.2)$ & \\
\hline II & 31 & $8(38.1)$ & $23(33.3)$ & \\
\hline III & 24 & $4(19.0)$ & $20(29.0)$ & \\
\hline IV & 26 & $5(23.8)$ & $21(30.4)$ & \\
\hline ECOG PS & & & & 0.098 \\
\hline $0-1$ & 68 & $19(90.5)$ & $49(73.1)$ & \\
\hline$\geq 2$ & 22 & $3(9.5)$ & $19(26.9)$ & \\
\hline LDH & & & & 0.346 \\
\hline Normal & 61 & $16(76.2)$ & $45(65.2)$ & \\
\hline$>$ Normal & 29 & $5(23.8)$ & $24(34.8)$ & \\
\hline $\begin{array}{l}\text { Extranodal } \\
\text { involvement }\end{array}$ & & & & 0.005 \\
\hline $0-1$ & 65 & $20(95.2)$ & $45(63.1)$ & \\
\hline$\geq 2$ & 25 & $1(4.8)$ & $24(36.9)$ & \\
\hline B symptom & & & & 0.606 \\
\hline No & 47 & $12(57.1)$ & $35(50.7)$ & \\
\hline Yes & 43 & $9(42.9)$ & $34(49.3)$ & \\
\hline IPI status & & & & 0.004 \\
\hline $0-1$ & 44 & $16(76.2)$ & $28(40.6)$ & \\
\hline$\geq 2$ & 46 & $5(23.8)$ & $41(59.4)$ & \\
\hline$\beta_{2}-\mathrm{MG}$ & & & & 0.233 \\
\hline Normal & 53 & $13(68.4)$ & $40(53.0)$ & \\
\hline$>$ Normal & 37 & 6 (31.6) & $31(47.0)$ & \\
\hline Treatment protocol & & & & 0.750 \\
\hline R-CHOP & 49 & $14(66.7)$ & $35(50.7)$ & \\
\hline $\mathrm{CHOP}$ & 41 & $7(33.3)$ & $34(49.3)$ & \\
\hline
\end{tabular}

GCB, germinal centre B-cell like; ECOG PS, Eastern Cooperative Oncology Group performance status; LDH, lactate dehydrogenase; IPI, International Prognostic Index; $\beta_{2}-\mathrm{MG}, \beta 2$ microglobulin.

regression analysis were carried out to assess progressionfree survival (PFS) times and overall survival (OS) time of the de novo DLBCL cases. P-value $<0.05$ was considered to denote statistical significance. Receiver operating characteristic (ROC) analysis was performed to determine an optimal cutoff value of miRNA expression $\left(2^{-\Delta \mathrm{Ct}}\right)$ to split the patients into a low-expression group and a high-expression group. PFS was calculated as the time of diagnosis to the date of clinical relapse, death or last contact. OS was defined as the date of diagnosis to death from any cause. Data were censored if the patients were alive at last follow-up. Mean follow-up time was 26.3 months (range 6-83). Curves were compared by univariate (log-rank) analysis using GRAPHPAD Prism version 4.00 ( $\mathrm{La}$ Jolla, CA, USA). 
A

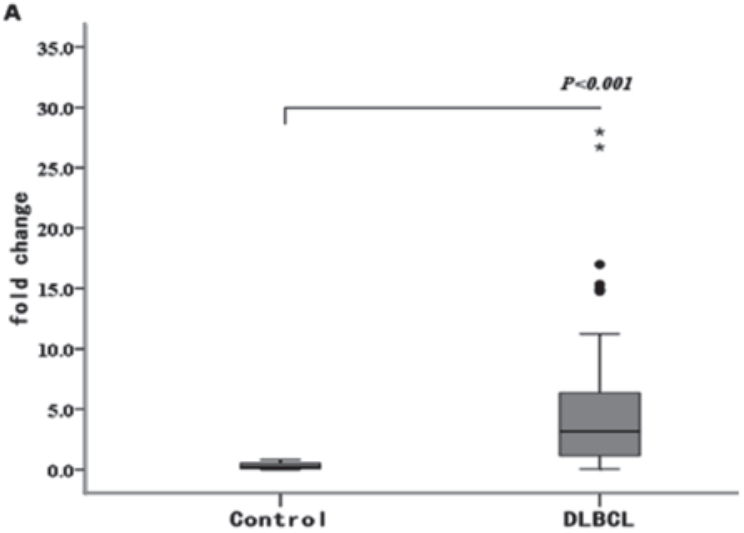

c
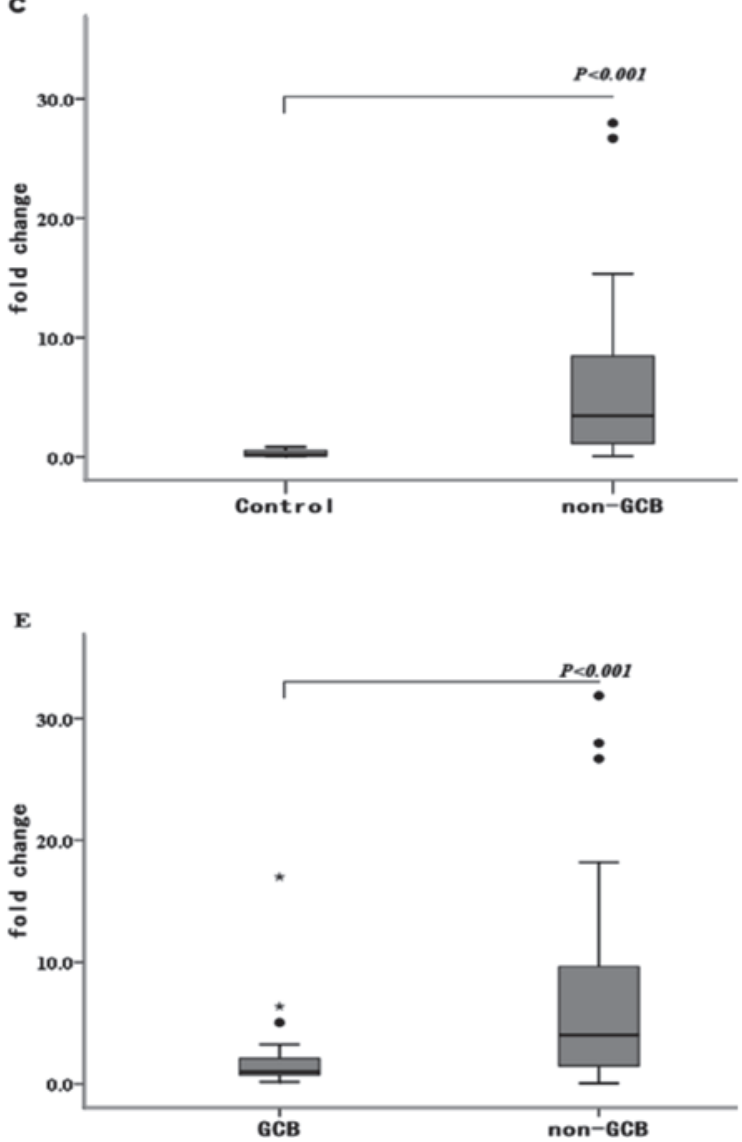

B

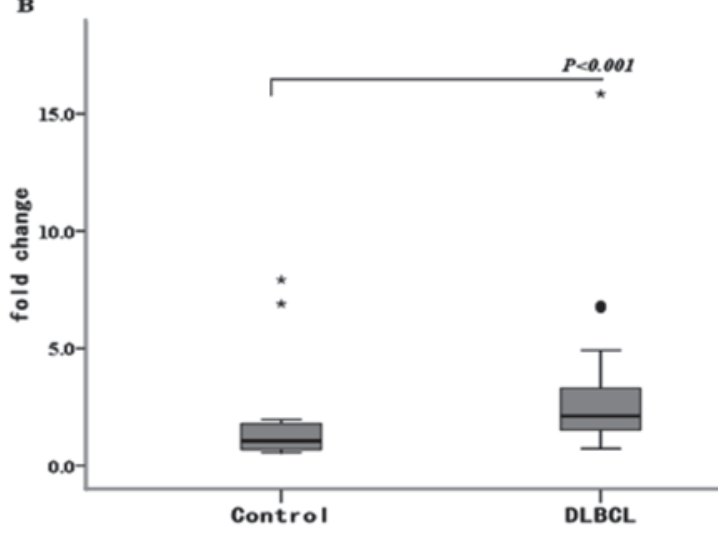

D
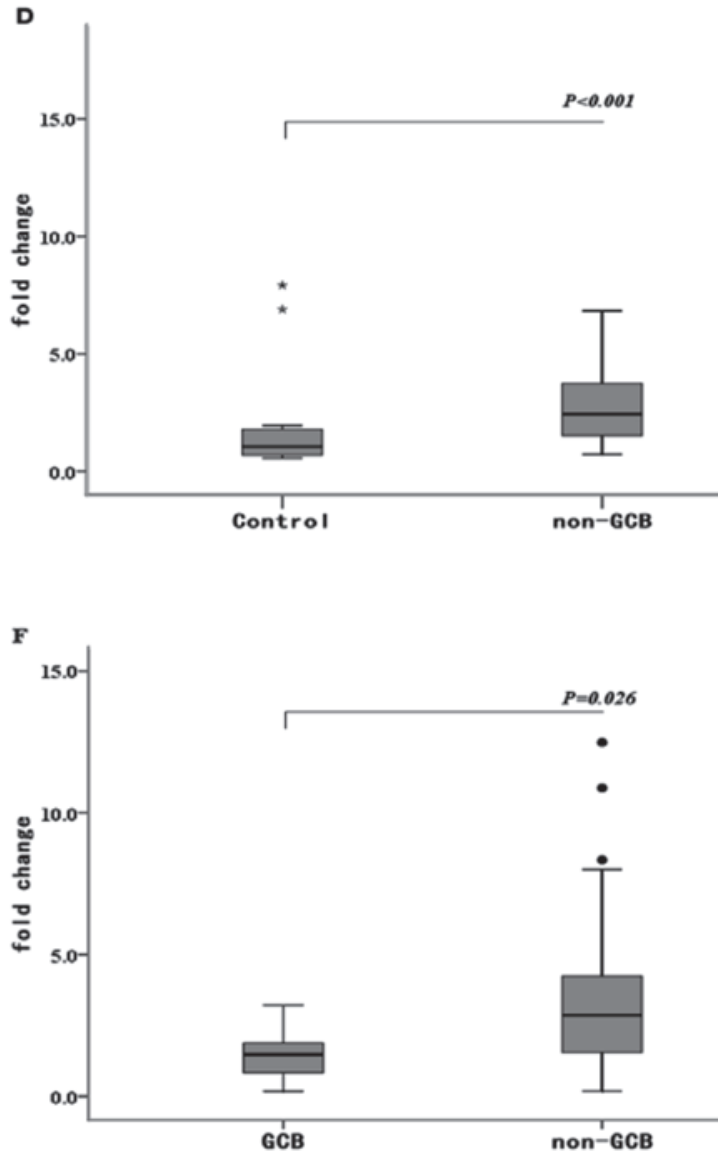

Figure 1. Expression levels of microRNAs in formalin-fixed/paraffin-embedded samples measured by RTQ-PCR. (A, C and E) miR-155; (B, D and F) miR-146a. (A-D) Fold change $\left(2^{-\Delta C}\right)$ is shown relative to controls. (E and F) Fold change is shown relative to germinal centre B cell-like lymphoma. P-values were calculated by the Mann-Whitney independent t-test.

\section{Results}

miR-155 and miR-146a expression levels of DLBCL patients are distinct from reactive hyperplasia lymphoid nodes and differ between $G C B$ and non-GCB subtypes of DLBCL. The expression levels of both tumor-associated miRNAs (miR-155 and miR-146a) in biopsy samples from DLBCL patients $(n=90)$ were compared to those of reactive hyperplasia lymphoid nodes $(n=31)$ by RTQ-PCR. The levels of both miRNAs were up-regulated in the DLBCL patients $(\mathrm{P}<0.001)$ (Fig. 1A and B), corresponding to an average fold-change of 18.97 and 2.46, respectively.
To ascertain whether the expression of miR-155 and miR146a is DLBCL immunophenotype-specific, we measured the expression levels of these miRNAs in 21 patients with GCB subtype DLBCL and in 69 patients with non-GCB subtype by RTQ-PCR. The expression levels of miR-155 and miR-146a were higher in the non-GCB DLBCL patients than in reactive hyperplasia lymphoid nodes $(\mathrm{P}<0.001)$ (Fig. 1C and D), corresponding to an average fold-change of 20.80 and 2.13 , respectively. We also found that the expression levels of miR-155 and miR-146a were higher in the non-GCB DLBCL patients compared to the GCB DLBCL patients, corresponding to an average fold-change of 1.58 

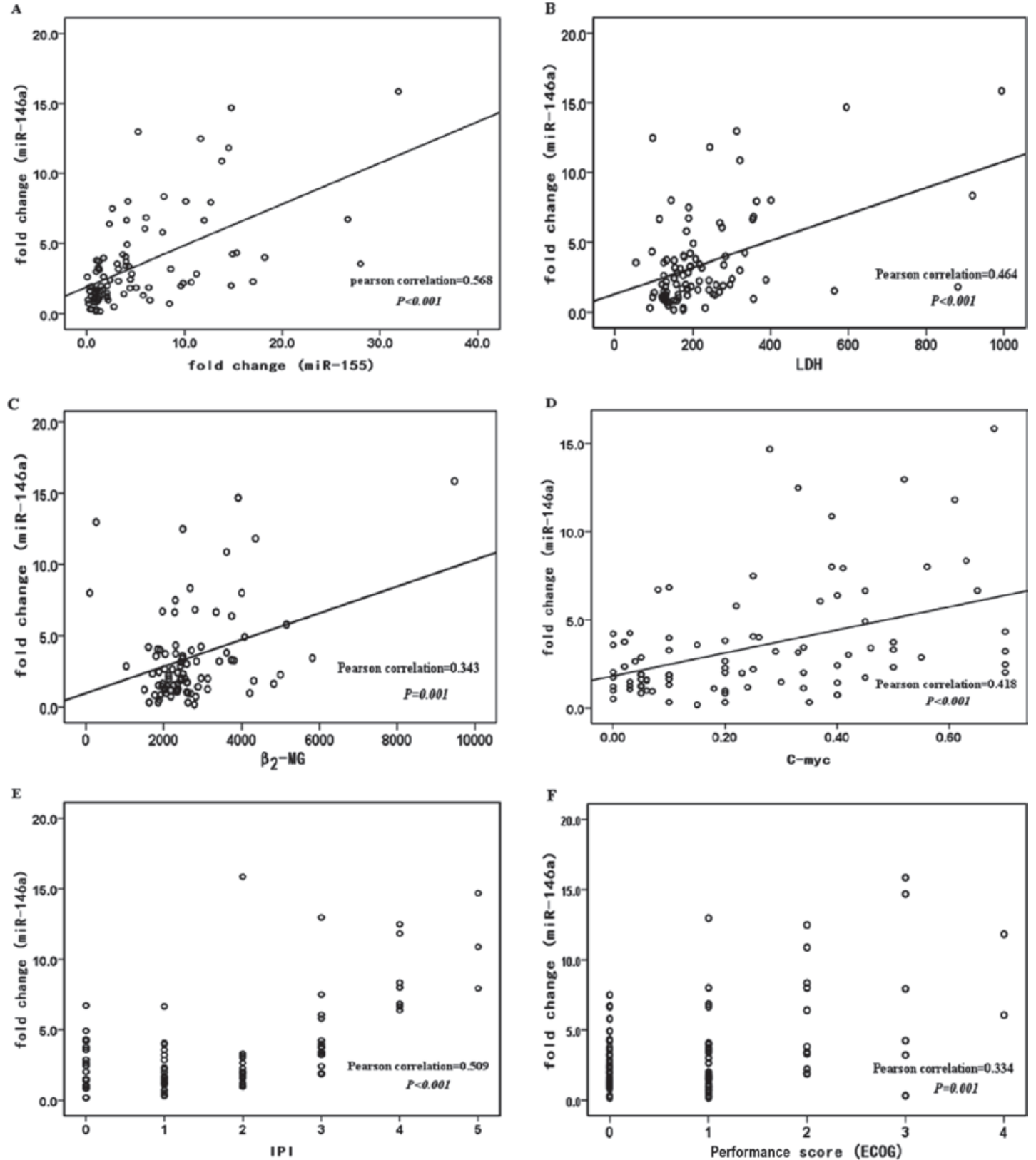

Figure 2. Expression levels of miR-146a are correlated with miR-155 and clinical patient characteristics in formalin-fixed/paraffin-embedded samples of DLBCL patients. Linear regression lines are shown. (A) miR-146a and miR-155, $\mathrm{r}^{2}=0.323, \mathrm{P}<0.001$; (B) miR-146a and LDH, $\mathrm{r}^{2}=0.215, \mathrm{P}<0.001$; (C) $\mathrm{miR}-146 \mathrm{a}$ and $\beta_{2}-\mathrm{MG}, \mathrm{r}^{2}=0.118, \mathrm{P}=0.001$; (D) miR-146a and $\mathrm{c}-\mathrm{myc}, \mathrm{r}^{2}=0.175, \mathrm{P}<0.001 ;$ (E) miR-146a and ECOG performance score, $\mathrm{r}^{2}=0.227, \mathrm{P}=0.001 ;(\mathrm{F}) \mathrm{miR}-146 \mathrm{a}$ and IPI status, $\mathrm{r}^{2}=0.337, \mathrm{P}<0.0018$.

and 1.69 , respectively $(\mathrm{P}<0.001$ and $\mathrm{P}=0.026$, respectively) (Fig. 1E and F).

miR-146a expression levels are associated with miR-155 expression levels and clinical characteristics in the de novo DLBCL patients. Clinical data, including lactate dehydrogenase (LDH), $\beta 2$ microglobulin $\left(\beta_{2}-\mathrm{MG}\right), \mathrm{c}-\mathrm{myc}$, International Prognostic Index (IPI) status and Eastern Cooperative Oncology Group physical score (ECOG PS), were available for 90 cases of the de novo DLBCL patients. The cases were split into two groups according to low IPI scores ( 0 and 1$)$ or high IPI scores (2, 3 and 4), and low ECOG PS (0 and 1) or high scores (2, 3 and 4). We found that miR-146a expression levels were associated with miR-155 expression levels $\left(\mathrm{r}^{2}=0.323, \mathrm{P}<0.001\right)$, LDH $\left(r^{2}=0.215, \mathrm{P}<0.001\right), \beta_{2}-\mathrm{MG}\left(\mathrm{r}^{2}=0.118, \mathrm{P}=0.001\right), \mathrm{c}-\mathrm{myc}$ $\left(\mathrm{r}^{2}=0.175, \mathrm{P}<0.001\right)$, IPI $\left(\mathrm{r}^{2}=0.337, \mathrm{P}<0.001\right)$ and ECOG PS $\left(r^{2}=0.227, P=0.001\right)$ in the same patients (Fig. 2A-F). miR146a expression levels showed no association with gender, age, clinical stage, B symptom and extranodal involvement. Meanwhile, there was no association between miR-155 expression levels and all the clinical characteristics mentioned above.

miR-155 and miR-146a expression is associated with clinical outcome in de novo DLBCL patients. The cutoff value, sensitivity and specificity of miR-155/miR-146a were 3.9840/2.0196, 80/88\% and 58.5/56.9\%, respectively (Fig. 3A and B). De novo DLBCL patients with low expression of miR-155 and miR-146a were found to be associated with a high complete remission (CR) rate (miR-155 76.5 vs. $43.8 \%$, $\mathrm{P}=0.032$; miR-146a 83.3 vs. $50 \%, \mathrm{P}=0.013$ ) and a high overall 

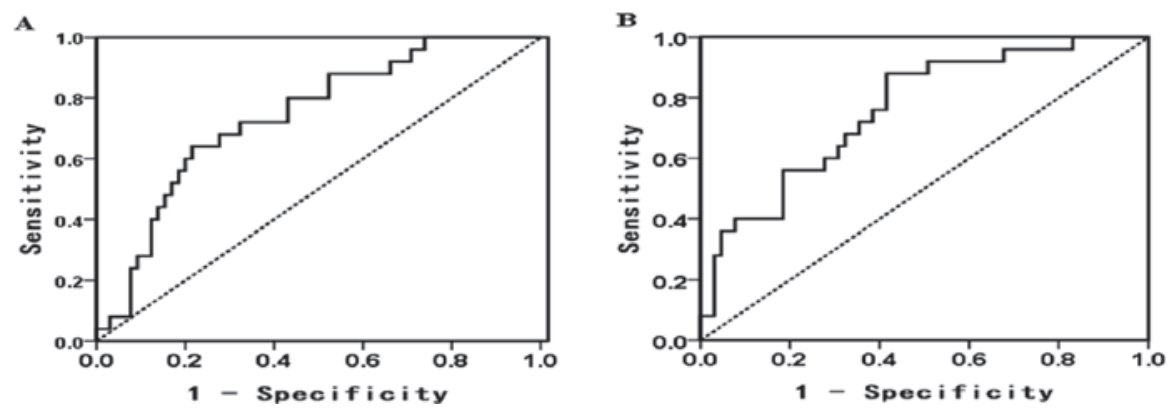

Figure 3. ROC curve of microRNA expression values $\left(2^{-\Delta C}\right.$ ). (A) miR-155, cutoff value 3.9840 (sensitivity $80 \%$; specificity $56.9 \%$; $95 \%$ CI $0.632-0.850$, $\mathrm{P}<0.001$ ). (B) miR-146a, cut-off value 2.0196 (sensitivity $88 \%$; specificity $58.5 \%$; $95 \%$ CI $0.655-0.867, \mathrm{P}<0.001$ ).
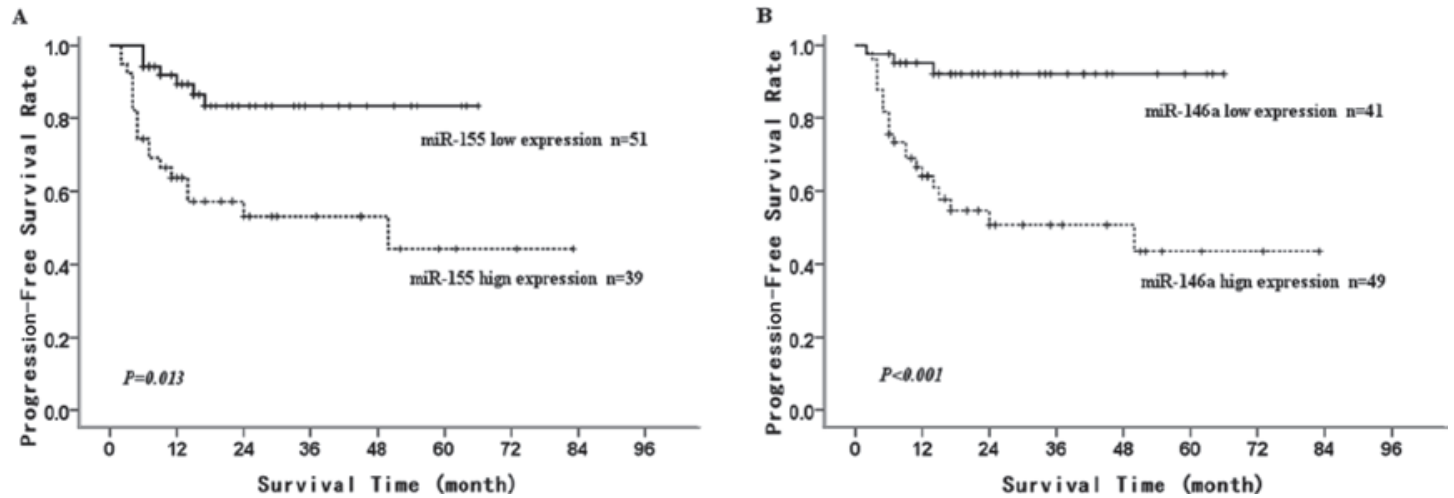

Figure 4. Kaplan-Meier survival curves were compared by univariate (log-rank) analysis and microRNA expression levels in patients were defined as high or low relative to the ROC cutoff value. (A) miR-155, $\mathrm{P}=0.013$; (B) miR-146a, $\mathrm{P}<0.001$.

Table II. Cox regression analysis of the prognostic factors associated with progression-free survival of the DLBCL patients.

\begin{tabular}{lccc}
\hline & $\begin{array}{c}\text { Cox regression } \\
\text { coefficient }\end{array}$ & $\begin{array}{c}\text { Hazard ratio } \\
(95 \% \mathrm{CI})\end{array}$ & P-value \\
\hline miR-155 & -1.345 & $0.260(0.085-0.801)$ & 0.019 \\
miR-146a & 0.113 & $1.119(0.977-1.282)$ & 0.104 \\
IPI status & -1.669 & $0.188(0.052-0.689)$ & 0.012
\end{tabular}

$\mathrm{P}<0$.05. DLBCL, diffuse large B-cell lymphoma; IPI, International Prognostic Index.

response (OR) rate (miR-155 85.3 vs. 50\%, $\mathrm{P}=0.008$; $\mathrm{miR}-146 \mathrm{a}$ 87.5 vs. $61.5 \%, \mathrm{P}=0.037$ ).

To assess the potential prognostic impact of these two miRNAs in de novo DLBCL cases, we performed a retrospective analysis. Using Kaplan-Meier survival (univariate) analysis, we found that low miR-155 and miR-146a expression was associated with a longer 5-year PFS in the de novo DLBCL cases $(83.3 \pm 5.9$ vs. $44.3 \pm 10.8 \%, \mathrm{P}=0.013 ; 92 \pm 4.4$ vs. $43.5 \pm 9.7 \%, \mathrm{P}<0.001$; Fig. $4 \mathrm{~A}$ and $\mathrm{B})$. We further examined the effect of various factors that may affect prognostic outcome in this cohort (i.e., gender, age, clinical stage, B symptom and extranodal involvement IPI stage and ECOG PS), as well as miRNA expression levels by multivariate Cox proportional hazard regression analysis. We found that the expression levels of miR-155 and IPI status, but not the expression levels of miR-146a, were statistically significant independent indicators of prognosis in this cohort $(\mathrm{P}<0.05)$ (Table II).

Expression of miR-155 is an independent indicator for chemotherapy protocol selection in the de novo DLBCL. Compared to $\mathrm{CHOP}$ protocol, the patients in the high miR-155 expression group who chose R-CHOP protocol for treatment achieved better 5-year OS and 5-year PFS (CHOP vs. RCHOP: 5-year OS 29.7 \pm 16.4 vs. 83.9 $\pm 8.5 \%, \mathrm{P}=0.035$; 5-year PFS 29.6 \pm 12.4 vs. $59.2 \pm 15.5 \%, \mathrm{P}=0.029$ ) (Fig. $5 \mathrm{~A}$ and $\mathrm{B}$ ). This difference was not noted in the low miR-155 expression group. miR-146a expression was not an indicator of chemotherapy protocol selection in the de novo DLBCL patients (data not shown).

\section{Discussion}

It is believed that $10-30 \%$ of all human genes are a target for miRNA regulation (19). Recent evidence shows that the expression of miRNA genes is deregulated in human cancer. miRNA overexpression may result in the down-regulation of tumorsuppressor genes, whereas their underexpression may lead to oncogene up-regulation (20-22). The potential importance of miRNAs in cancer is also implied by the finding that the majority of human miRNAs are located at cancer-associated genomic regions. Cancer-associated miRNAs have oncogenic properties (3). Moreover, it has been suggested that miRNA expression profiling may distinguish cancers according to diagnostic type and developmental stage of the tumor to a 

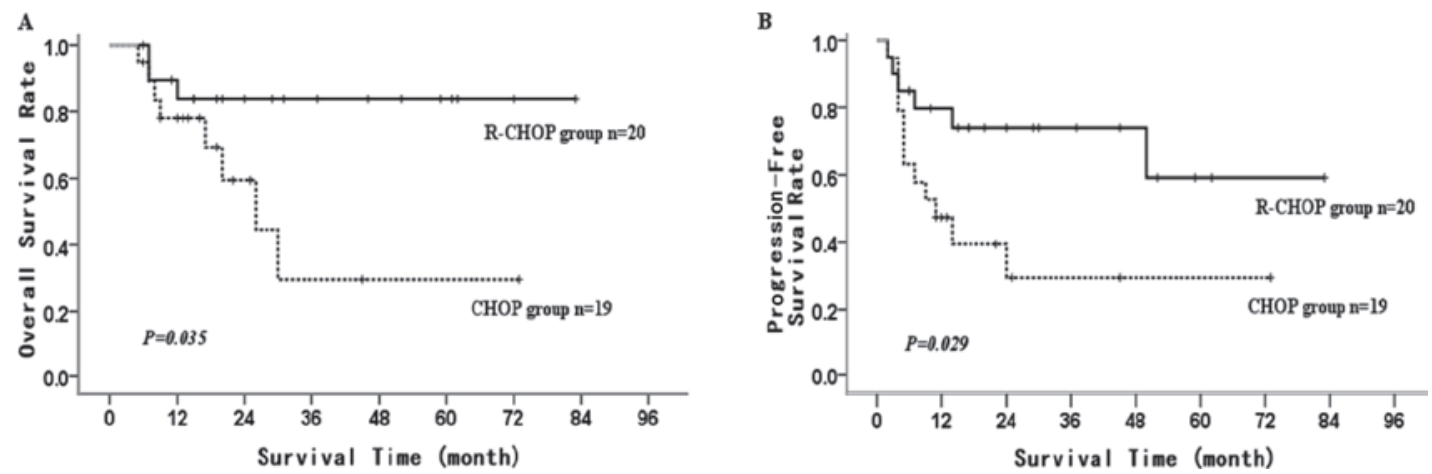

Figure 5. Kaplan-Meier survival curves were compared by univariate (log-rank) analysis, and treatment protocols in patients with high miR-155 expression levels were $\mathrm{CHOP}$ or R-CHOP. (A) Overall survival rate, $\mathrm{P}=0.035$; (B) Progression-free survival rate, $\mathrm{P}=0.029$.

greater degree of accuracy than traditional gene expression analysis (23). Although some progress has been made in identifying lymphoma-associated miRNAs by profiling a large number of lymphoma cell lines (24), clinical samples exhibit a discrete miRNA pattern compared to cell lines, reinforcing the need for studies on clinical material (25).

miR-155 and miR-146a as diagnostic tools in DLBCL patients. Seeking new tools for the diagnosis and management of lymphoma has been an ongoing endeavor. miRNAs due to their small size are relatively resistant to RNase degradation and, unlike mRNA, can be successfully recovered intact from archival FFPE material $(15,16,26)$. Furthermore, miRNAs can be detected in biological fluids, including serum and plasma (6). All of these make miRNAs appropriate candidates for lymphoma research.

Georgantas et al proposed that miR-155, miR-146a and another four microRNAs may act to prevent early-stage progenitor cells from differentiating to a more mature stage based on a bioinformatics approach (27). In this study, we found that miR-155 and miR-146a expression levels in DLBCL patient tissue were higher than levels in reactive hyperplasia lymphoid nodes. miR-155 expression has also been shown by other groups to be more highly expressed in DLBCL patients, particularly in patients with a non-GCB immunophenotype $(16,28,29)$. Yet, the research of miR-146a in lymphoma is limited. Down-regulation of miR-146a expression by the c-Myc oncogenic transcription factor has been previously observed in human and mouse B-cell lymphomas (30). Recent studies have evaluated the expression levels of miR-146a in acute lymphocytic leukemia and chronic lymphocytic leukemia (31-33). Their findings imply that miR-146a expression involved in deletion or amplification may be cell type-specific. The expression levels of miR-146a are not coincident among hematopoietic malignancies.

Differential expression of miR-155 and miR-146a between DLBCL patient lymphoid nodes and reactive hyperplasia lymphoid nodes suggest that miR-155 and miR-146a may be a potential tool for future molecular diagnostics in DLBCL.

miR-155 and miR-146a as prognostic biomarkers in DLBCL patients. Despite the fact that up to $80 \%$ of DLBCL patients reach a complete remission using a rituximab-based chemotherapy protocol, the response to treatment remains variable. A substantial proportion of DLBCL patients will still succumb to this disease (8). We sought to identify definite miRNAs that are associated with clinical outcome. Many studies have shown that miRNA expression levels have potential prognostic significance in malignant diseases, such as chronic lymphocytic leukemia, DLBCL, lung and pancreatic cancer $(5,16,23,34)$. We first examined whether miR-155 and miR-146a were associated with clinical prognostic factors, including $\mathrm{LDH}, \beta_{2}-\mathrm{MG}$, c-myc, IPI and ECOG PS. We found that the expression levels of miR-146a, but not miR-155, were associated with these prognostic factors. The significance of miR-155 expression is consistent with the findings of Lawrie et al $(6,16,25)$. We also found that the expression of miR-146a was correlated with miR-155. We know that the NF- $\mathrm{B}$ transcription factor activity is abnormal in DLBCL $(11,13)$. The phenomena also provide evidence that these two miRNAs may be regulated by the NF- $\kappa \mathrm{B}$ transcription factor $(12,14)$. Recently Volinia et al generated an Em/VH miR-155 transgenic mouse model. miR-146a expression levels were up-regulated in miR-155 transgenic mice when compared to the control wild-type mice. This suggests that miR-155 and miR-146a are involved in one complex microRNA network (35).

In order to ascertain whether miRNA expression is able to predict outcome, we analyzed PFS and OS in the DLBCL de novo patients. We found that low expression of miR-155 and miR-146a was associated with a high CR rate, a high OR rate and long PFS time. We carried out univariate analysis on the expression levels of these miRNAs individually and found that miR-155 and IPI were statistically significant independent prognostic indicators. Former studies indicate that miR-155 is an independent prognostic indicator only in non-GCBtype DLBCL patients $(25,28,29)$. We speculate that the high percentage of non-GCB patients in our group may have caused this discrepancy. Recently, Korean doctors found that NK/T cell lymphoma patients with low miR-146a expression had a significantly poor prognosis in the clinic. miR-146a was up-regulated in TH1 cells throughout murine hematopoietic development (36). p53/TA-p73/p63 increased the expression of c-myc-suppressed miR-146a in B-cell lymphogenesis (37). This seems to contradict our results. We believe that besides the different cell types, the effect of miRNAs on cell pathology and physiology is likely to be complex for at least two reasons: i) their activity is exerted in a one-to-many fashion, such that each miRNA controls the translation of tens or even hundreds of different coding messengers, and ii) a single messenger can be controlled by more than one miRNA. 
It is of note that a high expression level of miR-155, but not miR-146a, was an independent indicator for chemotherapy protocol selection in our study. Patients with high expression of miR-155 received more survival benefits from rituximab treatment. The reason is unclear. This may be related to inflammatory cytokines produced by constant activity of the $\mathrm{NF}-\kappa \mathrm{B}$ signaling pathway.

miR-155 and miR-146a as oncogenes in DLBCL patients. miRNAs, such as miR-155 and miR-146a, are associated with many types of cancer, including a wide range of solid and hematological malignancies (38). This phenomenon suggests that these miRNAs play a fundamental role in the establishment of a general malignant phenotype.

Thai et al used both BIC/miR-155-deficient and -knock-in mice to investigate the role of miR-155 in germinal centre (GC) $\mathrm{B}$-cell responses. They found a reduction in the total numbers of GCB cells in the deficient mice and an increase in numbers in the knock-in mice. In vitro activation of wild-type B cells resulted in strong up-regulation of miR-155 expression. Immunization resulted in decreased antibody titers in miR-155-deficient mice. It was also observed that activated miR-155-deficient B cells expressed about a third of the normal levels of TNF, suggesting that miR-155 controls B-cell activation through control of cytokine production (39). Transgenic mice expressing miR-155 targeted to B cells were also shown to spontaneously develop high-grade lymphomas (40). We found an association between the expression level of miR-155 and PFS in the studied patients; miR-155 was more highly expressed in all lymphoma cases, with an average expression 18.97-fold higher than the reactive hyperplasia lymphoid nodes.

Boldin et al suggested that miR-146a functions as a tumorsuppressor gene. They found that aging miR-146a-null mice developed frank tumors in lymphoid organs (41). This conclusion is in disagreement with our studies in DLBCL patients. We presume that miR-146a activity can be influenced either by the reposition of other genes close to its promoter regulatory regions (as c-myc translocation in lymphoma), or by the relocalization of other regulatory elements. The same gene, which behaves as an oncogene or suppressor gene, also depends on the transcriptional and/or post-transcriptional events.

In summary, our data suggest that miR-155 and miR146a may be diagnostic tools and prognostic indictors for DLBCL patients. Genetic associations have already provided compelling evidence that DLBCL subtypes represent discrete diseases that may arise by distinct pathogenetic pathways (42). Additional studies, including the characterization of miR-155 and miR-146a expression in a large number of DLBCL patients, are required to clarify the underlying basis for this association. Since miR-155 and miR-146a silence or modulate gene expression in humans through the regulation of transcription factors, an approach of targeted inhibition of miR-155 and miR-146a may be explored as a potential therapeutic target for controlling malignant lymphogenesis.

\section{Acknowledgements}

This study was supported by grants from the Shanghai Municipal Commission of Sciences and Technology fund (09ZR1418400), the Social Development fund of the Shanghai
Pudong District (PW2009D-5) and Key Discipline Project of Renji Hospital, Shanghai Jiaotong University School of Medicine (RJ 4101306). The authors thank Dr Qing-Wen Xie for the assistance in the statistical and survival analysis.

\section{References}

1. Chen K and Rajewsky N: The evolution of gene regulation by transcription factors and microRNAs. Nat Rev Genet 8: 93-103, 2007.

2. Kim VN: MicroRNA biogenesis: coordinated cropping and dicing. Nat Rev Mol Cell Biol 6: 376-385, 2005.

3. Calin GA, Sevignani C, Dumitru CD, et al: Human microRNA genes are frequently located at fragile sites and genomic regions involved in cancers. Proc Natl Acad Sci USA 101: 2999-3004, 2004.

4. Lawrie CH: MicroRNA expression in lymphoid malignancies: new hope for diagnosis and therapy? Cell Mol Med 12: 1432-1444, 2008.

5. Calin GA, Ferracin M, Cimmino A, et al: A microRNA signature associated with prognosis and progression in chronic lymphocytic leukemia. N Engl J Med 353: 1793-1801, 2005.

6. Lawrie CH, Gal S, Dunlop HM, et al: Detection of elevated levels of tumour associated microRNAs in serum of patients with diffuse large B-cell lymphoma. Br J Haematol 141: 672-675, 2008.

7. Kota SK and Balasubramanian S: Cancer therapy via modulation of microRNA levels: a promising future. Drug Discov Today 15: 733-740, 2010.

8. Coiffier B: Diffuse large cell lymphoma. Curr Opin Oncol 13: 325-334, 2001.

9. Alizadeh AA, Eisen MB, Davis RE, et al: Distinct types of diffuse large B-cell lymphoma identified by gene expression profiling. Nature 403: 503-511, 2000.

10. Hans CP, Weisenburger DD, Greiner TC, et al: Confirmation of the molecular classification of diffuse large B-cell lymphoma by immunohistochemistry using a tissue microarray. Blood 103: 275-282, 2004

11. Davis RE, Brown KD, Siebenlist U, et al: Constitutive nuclear factor kappaB activity is required for survival of activated B cell-like diffuse large B cell lymphoma cells. J Exp Med 194: 1861-1874, 2001.

12. Thompson RC, Herscovitch M, Zhao I, et al: NF-kappaB downregulates expression of the B-lymphoma marker CD10 through a miR-155/PU.1 pathway. J Biol Chem 286: 1675-1682, 2011.

13. Braun T, Carvalho G, Fabre C, et al: Targeting NF-kappaB in hematologic malignancies. Cell Death Differ 13: 748-758, 2006.

14. Taganov KD, Boldin MP, Chang KJ, et al: NF-kappaB dependent induction of microRNA miR-146, an inhibitor targeted to signaling proteins of innate immune responses. Proc Natl Acad Sci USA 103: 12481-12486, 2006.

15. Li J, Smyth P, Flavin R, et al: Comparison of miRNA expression patterns using total RNA extracted from matched samples of formalin-fixed paraffin-embedded (FFPE) cells and snap frozen cells. BMC Biotechnol 7: 36, 2007.

16. Lawrie CH, Soneji S, Marafioti T, et al: MicroRNA expression distinguishes between germinal center B cell-like and activated B cell-like subtypes of diffuse large B cell lymphoma. Int J Cancer 121: 1156-1161, 2007.

17. Nogai H, Dörken B and Lenz GJ: Pathogenesis of non-Hodgkin's lymphoma. Clin Oncol 29: 1803-1811, 2011.

18. Chen C, Ridzon DA, Broomer AJ, et al: Real-time quantificiation of microRNAs by stem-loop RT-PCR. Nucletic Acids Res 33: e179, 2005

19. Lewis BP, Burge CB and Bartel DP: Conserved seed pairing, often flanked by adenosines, indicates that thousands of human genes are microRNA targets. Cell 120: 15-20, 2005.

20. Croce CM and Calin GA: miRNAs, cancer, and stem cell division. Cell 122: 6-7, 2005.

21. Gregory RI and Shiekhattar R: MicroRNA biogenesis and cancer. Cancer Res 65: 3509-3512, 2005.

22. McManus MT: MicroRNAs and cancer. Semin Cancer Biol 13: 253-258, 2003.

23. Lu J, Getz G, Miska EA, et al: MicroRNA expression profiles classify human cancers. Nature 435: 834-838, 2005.

24. Lawrie CH, Saunders NJ, Soneji S, et al: MicroRNA expression in lymphocyte development and malignancy. Leukemia 22: 1440-1446, 2008. 
25. Lawrie $\mathrm{CH}$, Chi JX, Taylor S, et al: Expression of microRNAs in diffuse large B cell lymphoma is associated with immunophenotype, survival and transformation from follicular lymphoma. J Cell Mol Med 13: 1248-1260, 2009.

26. Xi Y, Nakajima G, Gavin E, et al: Systematic analysis of microRNA expression of RNA extracted from fresh frozen and formalin-fixed paraffin-embedded samples. RNA 13: 1668-1674, 2007.

27. Georgantas RW III, Hildreth R, Morisot S, et al: CD34+ hematopoietic stem-progenitor cell microRNA expression and function: a circuit diagram of differentiation control. Proc Natl Acad Sci USA 104: 2750-2755, 2007.

28. Eis PS, Tam W, Sun L, et al: Accumulation of miR-155 and BIC RNA in human B cell lymphomas. Proc Natl Acad Sci USA 102: 3627-3632, 2005.

29. Kluiver J, Poppema S, de Jong D, et al: BIC and miR-155 are highly expressed in Hodgkin, primary mediastinal and diffuse large B cell lymphomas. J Pathol 207: 243-249, 2005.

30. Chang TC, Yu D, Lee YS, et al: Widespread microRNA repression by Myc contributes to tumorigenesis. Nat Genet 40: 43-50, 2008.

31. Zhang $\mathrm{H}$, Luo XQ, Zhang P, et al: MicroRNA patterns associated with clinical prognostic parameters and CNS relapse prediction in pediatric acute leukemia. PLoS One 4: e7826, 2009.

32. Wang $\mathrm{Y}, \mathrm{Li} \mathrm{Z}, \mathrm{He} \mathrm{C}$, et al: MicroRNA expression signatures are associated with lineage and survival in acute leukemias. Blood Cells Mol Dis 44: 191-197, 2010.

33. Visone R, Rassenti LZ, Veronese A, et al: Karyotype-specific microRNA signature in chronic lymphocytic leukemia. Blood 114: 3872-3879, 2009.
34. Roldo C, Missiaglia E, Hagan JP, et al: MicroRNA expression abnormalities in pancreatic endocrine and acinar tumors are associated with distinctive pathologic features and clinical behavior. J Clin Oncol 24: 4677-4684, 2006.

35. Volinia S, Galasso M, Costinean S, et al: Reprogramming of miRNA networks in cancer and leukemia. Genome Res 20: 589-599, 2010.

36. Monticelli S, Ansel KM, Xiao C, et al: MicroRNA profiling of the murine hematopoietic system. Genome Biol 6: R71, 2005.

37. Boominathan L: The guardians of the genome (p53, TA-p73, and TA-p63) are regulators of tumor suppressor miRNA network. Cancer Metastasis Rev 29: 613-639, 2010.

38. Volinia S, Calin GA, Liu CG, et al: A microRNA expression signature of human solid tumors defines cancer gene targets. Proc Natl Acad Sci USA 103: 2257-2261, 2006.

39. Thai TH, Calado DP, Casola S, et al: Regulation of the germinal center response by microRNA-155. Science 316: 604-608, 2007.

40. Costinean S, Zanesi N, Pekarsky Y, et al: Pre-B cell proliferation and lymphoblastic leukemia/highgrade lymphoma in $\mathrm{E}$ (mu)-miR155 transgenic mice. Proc Natl Acad Sci USA 103: 7024-7029, 2006

41. Boldin MP, Taganov KD, Rao DS, et al: miR-146a is a significant brake on autoimmunity, myeloproliferation, and cancer in mice. J Exp Med 208: 1189-1201, 2011

42. Lenz G, Wright GW, Emre NCT, et al: Molecular subtypes of diffuse large B-cell lymphoma arise by distinct genetic pathways. Proc Natl Acad Sci USA 105: 13520-13525, 2008. 